\title{
Generalization of Stirling Number of the Second Kind and Combinatorial Identity
}

\author{
Ji Peng \\ Department of Electronic Information, Nanjing University, Nanjing, China \\ Email: mcfroo@sina.com
}

How to cite this paper: Peng, J. (2020) Generalization of Stirling Number of the Second Kind and Combinatorial Identity. Open Access Library Journal, 7: e6462. https://doi.org/10.4236/oalib.1106462

Received: May 26, 2020

Accepted: June 12, 2020

Published: June 15, 2020

Copyright $\odot 2020$ by author(s) and Open Access Library Inc.

This work is licensed under the Creative Commons Attribution International License (CC BY 4.0).

http://creativecommons.org/licenses/by/4.0/

\section{(c) (i) Open Access}

\section{Abstract}

The Stirling numbers of second kind and related problems are widely used in combinatorial mathematics and number theory, and there are a lot of research results. This article discuss the function: $\sum A_{1}^{C_{1}} A_{2}^{C_{2}} \cdots A_{k}^{C_{k}}$

$\left(C_{1}+C_{2}+\cdots+C_{K}=N-K, C_{i} \geq 0\right)$, obtain its calculation formula and a series of conclusions, which generalize the results of existing literature, and further obtain the combinatorial identity:

$\sum(-1)^{K-i} * C(K-1, K-i) C(A-1+i, N-1)=C(A, N-K)$.

\section{Subject Areas}

Combinatorics

\section{Keywords}

Combinatorics, Combinatorial Identity, Stirling Numbers, Calculation Formula

\section{Introduction}

Stirling number of the second kind $S_{2}(n, K)$ [1] is defined as

$$
t^{N}=\sum_{k=0}^{N} S_{2}(N, k)[t]_{k}
$$

It has attributes:

$$
\begin{aligned}
& \text { [1] } S_{2}(N, K)=\sum 1^{C_{1}} 2^{C_{2}} \cdots K^{C_{k}} \quad\left(C_{1}+C_{2}+\cdots+C_{K}=N-K, C_{i} \geq 0\right) \\
& \text { [1] } S_{2}(N, K)=\frac{1}{K !} \sum_{i=0}^{K-1}(-1)^{i}\left(\begin{array}{c}
K \\
i
\end{array}\right)(K-i)^{N} \\
& \text { Let } j=K-i \rightarrow S_{2}(N, K)=\frac{1}{(K-1) !} \sum_{j=1}^{K}(-1)^{K-j}\left(\begin{array}{c}
K-1 \\
K-j
\end{array}\right) j^{N-1}
\end{aligned}
$$


It is similar to the expansion of

$$
\begin{aligned}
& \quad(X-Y)^{N-1} \\
& S_{2}(0,0)=1, \quad S_{2}(N, 0)=0 \quad(N>0) \\
& S_{2}(N, 1)=1 \\
& S_{2}(N, 2)=\left(2^{N-1}-1^{N-1}\right) / 1 ! \\
& S_{2}(N, 3)=\left(3^{N-1}-2 * 2^{N-1}+1^{N-1}\right) / 2 ! \\
& S_{2}(N, 4)=\left(4^{N-1}-3 * 3^{N-1}+3 * 2^{N-1}-1^{N-1}\right) / 3 ! \\
& S_{2}(N, 5)=\left(5^{N-1}-4 * 4^{N-1}+6 * 3^{N-1}-4 * 2^{N-1}+1^{N-1}\right) / 4 ! \\
& S_{2}(N, 6)=\left(6^{N-1}-5 * 5^{N-1}+10 * 4^{N-1}-10 * 3^{N-1}+5 * 2^{N-1}-1^{N-1}\right) / 5 ! \\
& S_{2}(N, N-1)=\left(\begin{array}{c}
N \\
2
\end{array}\right)
\end{aligned}
$$

\section{Main Conclusion and Proof}

Definition: The generalization of Stirling number of the second kind

If $\{a\}=\left\{A_{1}, A_{2}, \cdots, A_{k}\right\}, A \in \mathbb{Z}, A_{i}<A_{j},(i<j)$, then

$$
\begin{gathered}
G(N, K,\{a\})=\sum A_{1}^{C_{1}} A_{2}^{C_{2}} \cdots A_{k}^{C_{k}} \quad\left(C_{1}+C_{2}+\cdots+C_{K}=N-K, C_{i} \geq 0\right) \\
G_{1}(N, K, A)=G(N, K,\{A, A+1, \cdots, A+K-1\}) \rightarrow S_{2}(N, K)=G_{1}(N, K, 1)
\end{gathered}
$$

The function has been discussed by many papers [2] [3] [4], including definition, recursive relation, generating function and so on. This article will not narrate.

1)

$$
\begin{aligned}
G(N, K,\{a\}) & =G\left(N-1, K-1,\left\{A_{1}, \cdots, A_{k-1}\right\}\right)+A_{k} * G(N-1, K,\{a\}) \\
& =G\left(N-1, K-1,\left\{A_{2}, \cdots, A_{k}\right\}\right)+A_{1} * G(N-1, K,\{a\})
\end{aligned}
$$

Proof: By definition.

The first equation corresponds to $S_{2}(n, K)=S_{2}(n-1, k-1)+k * S_{2}(n-1, K)$.

2) $G(N, K,\{a\})=\frac{G\left(N, K-1,\left\{A_{2}, \cdots, A_{k}\right\}\right)-G\left(N, K-1,\left\{A_{1}, \cdots, A_{k-1}\right\}\right)}{A_{k}-A_{1}}$

Proof: From the second equation of 1$)$.

3) $G(N, 1,\{A\})=A^{N-1}$, corresponds to $S_{2}(N, 1)=1$

4) $G\left(N, 2,\left\{A_{1}, A_{2}\right\}\right)=\frac{A_{2}^{N-1}-A_{1}^{N-1}}{A_{2}-A_{1}}=\frac{A_{1}^{N-1}}{A_{1}-A_{2}}+\frac{A_{2}^{N-1}}{A_{2}-A_{1}}$, corresponds to $S_{2}(N, 2)=2^{N-1}-1=2^{N-1}-1^{N-1}$.

Proof: $G\left(N, 2,\left\{A_{1}, A_{2}\right\}\right)=A_{1}^{N-2}+A_{1}^{N-3} A_{2}+\cdots+A_{2}^{N-2}$.

5) $G(N, K,\{a\})=\sum_{i=1}^{K} \frac{\left(A_{i}\right)^{N-1}}{\prod_{i \neq j}\left(A_{i}-A_{j}\right)}$, this is the calculation formula.

Proof: Induce by 2), 3), 4). 
The form is symmetrical, for example:

$G(N, 3,\{a\})=\frac{A_{1}^{N-1}}{\left(A_{1}-A_{2}\right)\left(A_{1}-A_{3}\right)}+\frac{A_{2}^{N-1}}{\left(A_{2}-A_{1}\right)\left(A_{2}-A_{3}\right)}+\frac{A_{3}^{N-1}}{\left(A_{3}-A_{1}\right)\left(A_{3}-A_{2}\right)}$

[2] obtains it by generating function.

Lemma 1: if $\{a\}$ is an equal difference sequence $\{A, A+d, \cdots, A+(K-1) d\}$, $\frac{1}{\prod_{i=m, i \neq j}\left(A_{i}-A_{j}\right)}=\frac{(-1)^{K-m}}{d^{K-1}(K-1) !}\left(\begin{array}{c}K-1 \\ K-m\end{array}\right)$.

Proof:

$$
\begin{aligned}
\frac{1}{\prod_{i=m, i \neq j}\left(A_{i}-A_{j}\right)} & =\frac{1}{\prod_{i=m, j<m}\left(A_{i}-A_{j}\right)} \frac{1}{\prod_{i=m, j>m}\left(A_{i}-A_{j}\right)} \\
& =\frac{1}{d^{K-1}} \frac{1}{(m-1) !} \frac{(-1)^{K-m}}{(K-m) !}
\end{aligned}
$$

$$
\begin{aligned}
& =\frac{(-1)^{K-m}(K-1) !}{d^{K-1}(K-1) !(m-1) !(k-m) !} \\
& =\frac{(-1)^{K-m}}{d^{K-1}(K-1) !}\left(\begin{array}{c}
K-1 \\
K-m
\end{array}\right)
\end{aligned}
$$

6) If $\{a\}=\{A, A+d, \cdots, A+(K-1) d\}$,

$G(N, K,\{a\})=\frac{1}{d^{K-1}(K-1) !} \sum_{j=1}^{K}(-1)^{K-j}\left(\begin{array}{l}K-1 \\ K-j\end{array}\right) A_{j}^{N-1}$.

Proof: By 5) and Lemma 1.

It is similar to the expansion of $(X-Y)^{N-1}$, in particular:

7) $G 1(N, K, A)=\frac{1}{(K-1) !} \sum_{i=1}^{K}(-1)^{K-i}\left(\begin{array}{c}K-1 \\ K-i\end{array}\right)(A-1+i)^{N-1} \quad$ similar to $\left(4^{\star}\right),\left(5^{\star}\right)$

8) $G_{1}(N, K, 1)=S_{2}(N, K)=\frac{1}{(K-1) !} \sum_{i=1}^{K}(-1)^{K-i}\left(\begin{array}{c}K-1 \\ K-i\end{array}\right) i^{N-1}$ equal to $\left(4^{*}\right),\left(5^{\star}\right)$

9) $G(N, K,\{d, 2 d, \cdots, K d\})=\frac{d^{N-K}}{(K-1) !} \sum_{i=1}^{K}(-1)^{K-i}\left(\begin{array}{c}K-1 \\ K-i\end{array}\right) i^{N-1}=d^{N-K} S_{2}(N, K)$

10) $G_{1}(K+1, K, A)=A+(A+1)+\cdots+(A+K-1)=K * A+\left(\begin{array}{c}K \\ 2\end{array}\right)$ corresponds to $\left(6^{*}\right)$

Theorem 1: $G_{1}(N<K, K,\{a\})=0 ; G_{1}(K, K,\{a\})=1$.

Proof:

7) $\rightarrow G_{1}(1, K \geq 1, A)=\frac{1}{(K-1) !} \sum_{i=1}^{K}(-1)^{K-i}\left(\begin{array}{l}K-1 \\ K-i\end{array}\right)=0$

4) $\rightarrow G_{1}(2,2, A)=1$

Suppose $G_{1}(X, K-1, A)$ match the theorem:

$$
\begin{aligned}
& G_{1}(N<K-1, K, A) \\
= & \frac{G_{1}\left(N, K-1,\left\{A_{2}, \cdots, A_{k}\right\}\right)-G_{1}\left(N, K-1,\left\{A_{1}, \cdots, A_{k-1}\right\}\right)}{A_{k}-A_{1}}=\frac{0-0}{K-1}=0
\end{aligned}
$$




$$
\begin{aligned}
& G_{1}(N=K-1, K, A) \\
& =\frac{G_{1}\left(N, K-1,\left\{A_{2}, \cdots, A_{k}\right\}\right)-G_{1}\left(N, K-1,\left\{A_{1}, \cdots, A_{k-1}\right\}\right)}{A_{k}-A_{1}}=\frac{1-1}{K-1}=0 \\
& \quad G_{1}(N=K, K, A) \\
& =\frac{G_{1}\left(N, K-1\left\{A_{2}, \cdots, A_{k}\right\}\right)-G_{1}\left(N, K-1,\left\{A_{1}, \cdots, A_{k-1}\right\}\right)}{A_{k}-A_{1}} \\
& =\frac{(K-1)(A+1)+\left(\begin{array}{c}
K \\
2
\end{array}\right)-(K-1) * A-\left(\begin{array}{c}
K \\
2
\end{array}\right)}{K-1}=1
\end{aligned}
$$

Induction proved.

q.e.d.

The theorem verify the definition, $A$ can be any integer.

Definition: $A \in Z, G_{2}(N, K, A)=\sum_{i=1}^{K}(-1)^{K-i}\left(\begin{array}{c}K-1 \\ K-i\end{array}\right)\left(\begin{array}{c}A-1+i \\ N-1\end{array}\right)$

Theorem 2: $G_{2}(N+K, K, A)=\left(\begin{array}{l}A \\ N\end{array}\right)$

\section{Proof:}

Let $F(N)=(N-1) ! G_{2}(N, K, A)=\sum_{i=1}^{K}(-1)^{K-i}\left(\begin{array}{l}K-1 \\ K-i\end{array}\right)[A-1+i]_{N-1}$.

Substitution $\left(1^{\star}\right)$ to 7$)$, use Theorem 1 :

$$
\begin{gathered}
G_{1}(1, K, A)=0, K>1 \rightarrow F(1)=0 \rightarrow G_{2}(1, K>1, A)=0 \\
G_{1}(2, K, A)=0, K>2, F(1)=0 \rightarrow F(2)=0 \rightarrow G_{2}(2, K>2, A)=0 \\
\ldots \\
G_{1}(K, K,\{a\})=1 \rightarrow F(K)=(K-1) ! \rightarrow \quad G_{2}(K, K, A)=1=\left(\begin{array}{c}
A \\
0
\end{array}\right)
\end{gathered}
$$

10) $\rightarrow$

$G_{1}(1+K, K, A)=K * A+\left(\begin{array}{c}K \\ 2\end{array}\right)=\frac{S_{2}(K, K) F(K+1)+S_{2}(K, K-1) F(K)}{(K-1) !} \rightarrow$

$F(1+K)=A * K ! \rightarrow G_{2}(1+K, K, A)=A=\left(\begin{array}{c}A \\ 1\end{array}\right)$

$\left(\begin{array}{c}A \\ N+1\end{array}\right)=\left(\begin{array}{c}A-1 \\ N\end{array}\right)+\left(\begin{array}{c}A-1 \\ N+1\end{array}\right) \rightarrow$

$G_{2}(N+1+K, K, A)=G_{2}(N+K, K, A-1)+G_{2}(N+1+K, K, A-1) \rightarrow$

$G_{2}(N+K, K, A)=\left(\begin{array}{l}A \\ N\end{array}\right)$

q.e.d.

Record in [5]:

$$
\sum_{k=0}^{m-1}(-1)^{k}\left(\begin{array}{l}
m \\
k
\end{array}\right)\left(\begin{array}{c}
m+n-k-1 \\
n
\end{array}\right)=\left(\begin{array}{c}
n-1 \\
m-1
\end{array}\right)
$$


Let $A=n-1, m=K-1, i=K-k \rightarrow$ left $=\sum_{i=0}^{K}(-1)^{K-i}\left(\begin{array}{c}K-1 \\ K-i\end{array}\right)\left(\begin{array}{c}A-1+i \\ A+1\end{array}\right)$.

Let $K+N-1=A+1 \rightarrow$

left $=\sum_{i=0}^{K}(-1)^{K-i}\left(\begin{array}{l}K-1 \\ K-i\end{array}\right)\left(\begin{array}{c}A-1+i \\ K+N-1\end{array}\right)=\left(\begin{array}{c}n-1 \\ m-1\end{array}\right)=\left(\begin{array}{c}A \\ A-N\end{array}\right)=\left(\begin{array}{l}A \\ N\end{array}\right)$.

$\left.{ }^{* *}\right)$ has 2 variables $(m, n)$, it is $G_{2}(K+A+2, K, A)$ actually.

Theorem 2 has 3 variables, is promotion of $\left.{ }^{* *}\right)$.

11) $G_{2}(N+K, K, A)$ : The Inclusion-Exclusion Principle.

$G_{2}(N+K, K, A)=\left(\begin{array}{l}A \\ N\end{array}\right)$ is independent of $K$.

Choice $N$ from $A$, one way is:

$$
\begin{aligned}
& \left(\begin{array}{l}
A \\
N
\end{array}\right)=\left(\begin{array}{c}
A-1 \\
N-1
\end{array}\right)+\left(\begin{array}{c}
A-1 \\
N
\end{array}\right)=\left(\begin{array}{c}
A-1 \\
N-1
\end{array}\right)+\left(\begin{array}{c}
A-2 \\
N-1
\end{array}\right)+\cdots+\left(\begin{array}{l}
N-1 \\
N-1
\end{array}\right) \\
& \left(\begin{array}{l}
A \\
N
\end{array}\right)=\left(\begin{array}{l}
A+1 \\
N+1
\end{array}\right)-\left(\begin{array}{c}
A \\
N+1
\end{array}\right)=G_{2}(N+2,2, A) \\
& \text { Another way is: } \quad=\left\{\left(\begin{array}{c}
A+2 \\
N+2
\end{array}\right)-\left(\begin{array}{c}
A+1 \\
N+2
\end{array}\right)\right\}-\left\{\left(\begin{array}{c}
A+1 \\
N+2
\end{array}\right)-\left(\begin{array}{c}
A \\
N+2
\end{array}\right)\right\} \\
& =G_{2}(N+3,3, A) \\
& G(N+K, K,\{a\})-\left(\sum A_{i}\right) G(N+K-1, K,\{a\}) \\
& \text { 12) }+\left(\sum A_{i} A_{j}\right) G(N+K-1, K,\{a\})+\cdots \\
& +(-1)^{K}\left(A_{1} A_{2} \cdots A_{k}\right) G(N, K,\{a\})=0
\end{aligned}
$$

\section{Proof:}

$$
\begin{aligned}
0 & =G_{1}(N, 0,\{a\})=G(N+1,1,\{a\})-A_{1} G(N, 1,\{a\}) \\
& =\left\{G(N+2,2, A)-A_{2} G_{1}(N+1,2, A)\right\}-A_{1}\left\{G(N+1,2, A)-A_{2} G_{1}(N, 2, A)\right\} \\
& =G(N+2,2, A)-\left(A_{1}+A_{2}\right) G_{1}(N+1,2, A)+A_{1} A_{2} G_{1}(N, 2, A)
\end{aligned}
$$

Induction proved.

q.e.d.

This is similar to the Inclusion-Exclusion Principle,in particular:

13)

$S_{2}(N, K)-S_{1}(K+1, K) S_{2}(N-1, K)+\cdots+(-1)^{K} S_{1}(K+1,1) S_{2}(N-K, K)=0$

$S_{1}$ is unsigned Stirling number of the first kind.

14) $G_{1}(N, K, A)=\sum_{t=K-1}^{N-1} S_{2}(N-1, t)\left(\begin{array}{c}A \\ t+1-K\end{array}\right)[K]^{t+1-K}$

\section{Proof:}

Substitution $\left(1^{\star}\right)$ to 7 ), use Theorem 2: 


$$
\begin{aligned}
G_{1}(N, K, A) & =\frac{1}{(K-1) !} \sum_{i=1}^{K}(-1)^{K-i}\left(\begin{array}{c}
K-1 \\
K-i
\end{array}\right)(A-1+i)^{N-1} \\
& =\frac{1}{(K-1) !} \sum_{i=1}^{K}(-1)^{K-i}\left(\begin{array}{c}
K-1 \\
K-i
\end{array}\right) \sum_{t=0}^{N-1} S_{2}(N-1, t)[A-1+i]_{t} \\
& =\sum_{t=0}^{N-1} S_{2}(N-1, t) \sum_{i=1}^{K}(-1)^{K-i}\left(\begin{array}{c}
K-1 \\
K-i
\end{array}\right)\left(\begin{array}{c}
A-1+i \\
t
\end{array}\right) \frac{t !}{(K-1) !} \\
& =\sum_{t=0}^{N-1} S_{2}(N-1, t) G_{2}(t+1, K, A)[K]^{t+1-K} \\
& =\sum_{t=0}^{N-1} S_{2}(N-1, t)\left(\begin{array}{c}
A \\
t+1-K
\end{array}\right)[K]^{t+1-K}
\end{aligned}
$$

q.e.d.

$\rightarrow S_{2}(N, K)=G_{1}(N, K, 1)=K * S_{2}(N-1, K)+S_{2}(N-1, K-1)$

\section{Conclusions}

This paper starting from $\left(4^{\star}\right),\left(5^{\star}\right)$, discusses the problems from different perspectives.

The introduced function has good characteristics, can be further studied.

\section{Conflicts of Interest}

The author declares no conflicts of interest regarding the publication of this paper.

\section{References}

[1] Hardy, G.H. (2010) An Introduction to the Theory of Numbers. 6th Edition. Zhang, F. Translation. People's Post and Telecommunications Press, Beijing.

[2] Li, C.X. (1995) The Expression of Stirling Number of the Second Kind and Others. Journal of Hubei Normal University: Philosophy and Social Sciences, 6, 72-76.

[3] Huang, R.H. and Chen, B.E. (2011) Some Conclusions on the Generalized Stirling Number of the Second Kind. Journal of Hanshan Normal University, 3, 29-31.

[4] Zhang, F.L. (2011) An Identity of Stirling Numbers of the Second Kind. Journal of Weinan Teachers College, 12, 14-16.

[5] Editorial Board of Handbook of Modern Applied Mathematics (2002) Handbook of Modern Applied Mathematics Discrete Mathematics Volume. Tsinghua University Press, Beijing. 